\title{
The Centre of the LMC Bar - Analysis of HST Data
}

\author{
P. Linde, A. Ardeberg \\ Lund Observatory, Box $43, S E-22100$ Lund, Sweden
}

B. Gustafsson

Uppsala Astronomical Observatory, Box 515, S-751 20 Uppsala, Sweden

\begin{abstract}
We used the HST to obtain high resolution images of the LMC Bar centre. Through careful data analysis, taking into account cleaning, dithering and crowded field decomposition, we show that accurate uvby photometry can be achieved for $\mathrm{F}$ dwarfs, reaching stars around the lower turn-off region, as faint as $\mathrm{V}=22$.
\end{abstract}

\section{Introduction}

To resolve individual main sequence stars in the core of the LMC Bar, we have used a total of 14 hours of exposure with the Hubble Space Telescope (HST) WFPC2 uvby filters. This allowed high precision photometry of F-G stars with $19.5<\mathrm{V}<22.5$. Additionally, stars down to $\mathrm{V}=26$ were included for luminosity function studies. Ardeberg et al. (1997) compares ground and space data, emphasising the necessity of high resolution observations for detailed LMC studies. To preserve the inherent high quality of the HST data, dedicated image analysis is necessary.

\section{Cosmic Ray Image Deteroriation and Image Dithering}

The space environment of the HST strongly affects long exposures, due to numerous impacts of charged particles on the CCD detectors. Efficient detection and elimination of these events are fundamental for good photometry. Fig. 1 (upper left) shows an example of detected impacts (no stars). With a modified median-filtering algorithm on several separate images of the same field, successful removal of the cosmic rays is possible.

Due to the under-sampling in WFPC2 images, accurate photometry is difficult in crowded fields, especially with the WF cameras. Through application of image dithering, it is possible to decrease this problem. We designed a dithering pattern such that each of 4 consecutive exposures were offset by a quarter of a pixel, simultaneously in $\mathrm{x}$ and $\mathrm{y}$ directions for both the PC and the WFC. Subsequent image restoration gives a significantly smaller apparent pixel size.

In Fig. 1, the lower left panel shows a sub field (from a single exposure) before filtering, the lower right panel after cleaning, dithering and summation of 16 exposures. The upper right panel shows the final full field. 

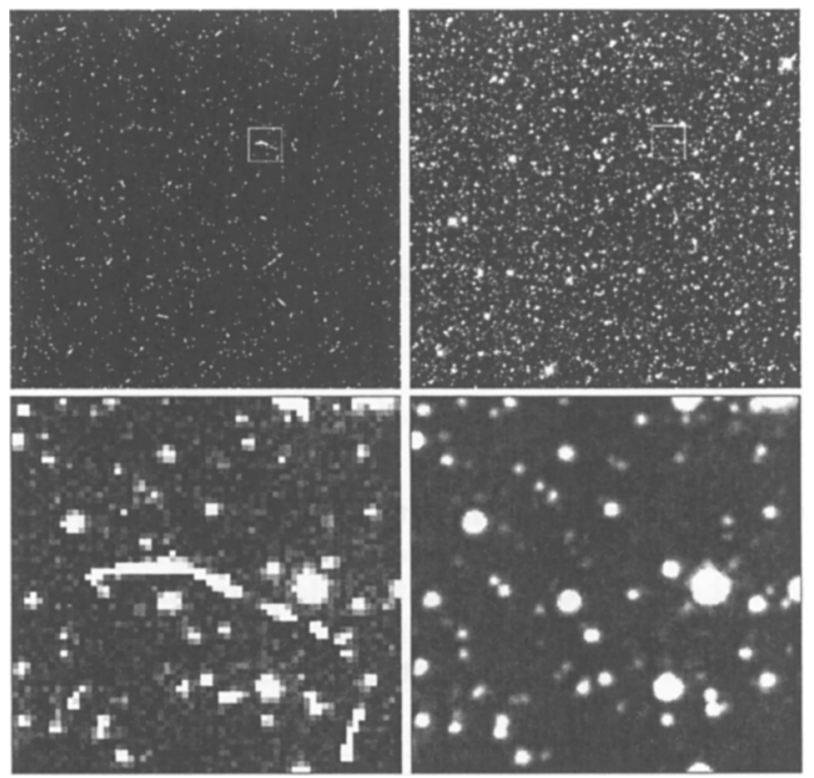

Figure 1. Upper left: Detected cosmic ray events in a single $800 \mathrm{sec}$ WF3 exposure. Upper right: Complete WF3 b field. Lower left: Detail from single WF3 b exposure. Lower right: Same field after filtering, dithering and adding 16 exposures.

\section{Photometric Performance}

$\mathrm{PC}$ and WFC photometric accuracy for our field is shown in Fig. 2. To evaluate photometric improvement from dithering, 16 PC images from the same field have been analysed, both as a set of 16 individual images and as four sets of dithered images. The accuracy is significantly improved (see Linde and Snel, 1997).

\section{References}

Ardeberg, A., Gustafsson, B., Linde, P., \& Nissen, P.-E., 1997, A\&A, 322, L13 Linde, P., \& Snel, R. 1997, ADASS VI, A.S.P. Conference Series, Vol. 125, eds. Gareth Hunt and H. E. Payne, p. 431
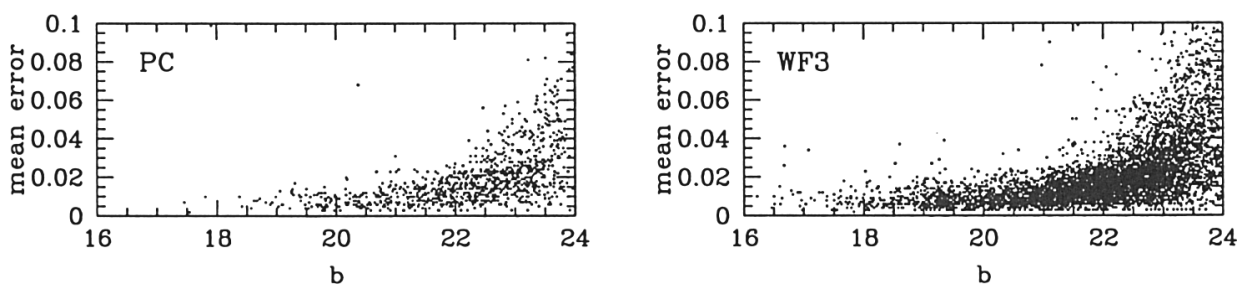

Figure 2. Mean error as function of $\mathrm{b}$ for the PC and the WF3. 